DOI: http://doi.org/10.21857/mwo1vczo8y

\title{
CODES FROM ORBIT MATRICES OF STRONGLY REGULAR GRAPHS
}

\author{
Dean Crnković, Marija Maksimović and Sanja Rukavina
}

\begin{abstract}
We show that under certain conditions submatrices of orbit matrices of strongly regular graphs span self-orthogonal codes. In order to demonstrate this method of construction, we construct self-orthogonal binary linear codes from orbit matrices of the triangular graphs $T(2 k)$ with at most 120 vertices. Further, we obtain strongly regular graphs and block designs from codewords of the constructed codes.
\end{abstract}

\section{INTRODUCTION}

We present a method for constructing self-orthogonal codes from submatrices of orbit matrices of strongly regular graphs. Applying this method we construct self-orthogonal binary linear codes from orbit matrices of triangular graphs $T(2 k), k=3,4,5,6,7,8$. The method of constructing self-orthogonal codes presented in this paper is a generalization of the method presented in [8]. We use the constructed codes to obtain strongly regular graphs and block designs. More precisely, the strongly regular graphs and block designs are constructed from codewords of a given weight of the obtained binary linear codes.

The paper is organized as follows: after a brief description of the terminology and some background results in Section 2, in Section 3 we describe the concept of orbit matrices of strongly regular graphs, based on results presented in $[2,8]$. In Section 4 we present a method for construction of self-orthogonal codes from orbit matrices of strongly regular graphs, and in Section 5 we apply this method to construct self-orthogonal codes from triangular graphs. In Section 6 we construct strongly regular graphs and BIBDs from codewords of the obtained codes.

2010 Mathematics Subject Classification. 05E30, 20D45, 94B05.

Key words and phrases. Strongly regular graph, self-orthogonal code, block design. 


\section{BACKGROUND AND TERMINOLOGY}

We assume that the reader is familiar with basic notions from the theory of finite groups. For basic definitions and properties of strongly regular graphs we refer the reader to [3] or [16].

A graph is regular if all its vertices have the same valency; a simple regular graph $\Gamma=(\mathcal{V}, \mathcal{E})$ is strongly regular with parameters $(v, k, \lambda, \mu)$ if it has $|\mathcal{V}|=v$ vertices, valency $k$, and if any two adjacent vertices are together adjacent to $\lambda$ vertices, while any two nonadjacent vertices are together adjacent to $\mu$ vertices. A strongly regular graph with parameters $(v, k, \lambda, \mu)$ is usually denoted by $\operatorname{SRG}(v, k, \lambda, \mu)$. An automorphism of a strongly regular graph $\Gamma$ is a permutation of vertices of $\Gamma$, such that every two vertices are adjacent if and only if their images are adjacent.

The triangular graph $T(n)$ is the line graph of the complete graph $K_{n}$. Triangular graphs $T(n)$ are strongly regular with parameters $(v, k, \lambda, \mu)=$ $(n(n-1) / 2,2(n-2), n-2,4)$.

An incidence structure $\mathcal{D}=(\mathcal{P}, \mathcal{B}, I)$, with point set $\mathcal{P}$, block set $\mathcal{B}$ and incidence $I \subseteq \mathcal{P} \times \mathcal{B}$, is a $2-(v, b, r, k, \lambda)$ design, if $|\mathcal{P}|=v,|\mathcal{B}|=b$, every block $B \in \mathcal{B}$ is incident with precisely $k$ points, every 2 distinct points are together incident with precisely $\lambda$ blocks and every point is incident with exactly $r$ blocks. If $b<\left(\begin{array}{l}v \\ k\end{array}\right)$, then $\mathcal{D}$ is called a balanced incomplete block design (BIBD).

A linear $q$-ary $(n, k)$ code $C$ over the finite field $F_{q}$ of prime-power order $q$ is a $k$-dimensional subspace of the $n$-dimensional vector space over $F_{q}$. The weight of a codeword is the number of its elements that are nonzero and the distance between two codewords is the Hamming distance between them, that is, the number of elements in which they differ. The minimum distance between distinct codewords is denoted by $d$. The minimum distance of a linear code is the minimum weight of its nonzero codewords. If a linear code $C$ over a field of order $q$ is of length $n$, dimension $k$, and minimum distance $d=d(C)$, then we write $[n, k, d]_{q}$ to show this information. An $[n, k]$ linear code $C$ is said to be a best known linear $[n, k]$ code if $C$ has the highest minimum weight among all known $[n, k]$ linear codes. An $[n, k]$ linear code $C$ is said to be an optimal linear $[n, k]$ code if the minimum weight of $C$ achieves the theoretical upper bound on the minimum weight of $[n, k]$ linear codes, and near-optimal if its minimum distance is at most 1 less than the largest possible value. The dual code $C^{\perp}$ is the orthogonal complement under the standard inner product $(\cdot, \cdot)$, i.e. $C^{\perp}=\left\{v \in F^{n} \mid(v, c)=0\right.$ for all $\left.c \in C\right\}$. If $C \subset C^{\perp}$, then $C$ is called self-orthogonal.

The support of a nonzero codeword $x=\left(x_{1}, \ldots, x_{n}\right)$ is the set of indices of its nonzero coordinates, i.e. $\operatorname{supp}(x)=\left\{i \mid x_{i} \neq 0\right\}$. The support design of a code of length $n$ for a given nonzero weight $w$ is the design with points the $n$ coordinate indices and blocks the supports of all codewords of weight $w$. 


\section{ORBIT MATRICES OF STRONGLY REGULAR GRAPHS}

Orbit matrices of block designs have been frequently used for construction of block designs with a presumed automorphism group, see e.g. [7] and [13]. In 2011 Behbahani and Lam introduced the concept of orbit matrices of SRGs (see [2]). While Behbahani and Lam were mostly focused on orbit matrices of strongly regular graphs admitting an automorphism of prime order, a general definition of an orbit matrix of a strongly regular graph is given in [8].

Let $\Gamma$ be a $\operatorname{SRG}(v, k, \lambda, \mu)$ and $A$ be its adjacency matrix. Suppose an automorphism group $G$ of $\Gamma$ partitions the set of vertices $V$ into $b$ orbits $O_{1}, \ldots, O_{b}$, with lengths $n_{1}, \ldots, n_{b}$, respectively. The orbits divide $A$ into submatrices $\left[A_{i j}\right]$, where $A_{i j}$ is the adjacency matrix of vertices in $O_{i}$ versus those in $O_{j}$. We define matrices $C=\left[c_{i j}\right]$ and $R=\left[r_{i j}\right], 1 \leq i, j \leq b$, such that

$$
\begin{aligned}
& c_{i j}=\text { column sum of } A_{i j}, \\
& r_{i j}=\text { row sum of } A_{i j} .
\end{aligned}
$$

The matrix $R$ is related to $C$ by

$$
r_{i j} n_{i}=c_{i j} n_{j}
$$

Since the adjacency matrix is symmetric, it follows that

$$
R=C^{T} .
$$

The matrix $R$ is the row orbit matrix of the graph $\Gamma$ with respect to $G$, and the matrix $C$ is the column orbit matrix of the graph $\Gamma$ with respect to $G$. The matrices $C=\left[c_{i j}\right]$ and $R=\left[r_{i j}\right]$ satisfy the following conditions (see [8]):

$$
\sum_{s=1}^{b} \frac{n_{s}}{n_{j}} c_{i s} c_{j s}=\delta_{i j}(k-\mu)+\mu n_{i}+(\lambda-\mu) c_{i j}
$$

and

$$
\sum_{s=1}^{b} \frac{n_{s}}{n_{j}} r_{s i} r_{s j}=\delta_{i j}(k-\mu)+\mu n_{i}+(\lambda-\mu) r_{j i}
$$

Let us assume that a group $G$ acts as an automorphism group of a $\operatorname{SRG}(v, k, \lambda, \mu)$. In order to enable a construction of SRGs with presumed automorphism group $G$, each matrix with the properties of a matrix $R$ or $C$ will be called a row orbit matrix or a column orbit matrix, respectively, for parameters $(v, k, \lambda, \mu)$ and a group $G$ (see [2]). Therefore, the following definition of orbit matrices of strongly regular graphs was introduced in [8].

Definition 3.1. $A(b \times b)$-matrix $R=\left[r_{i j}\right]$ with entries satisfying conditions: 


$$
\begin{aligned}
& \sum_{j=1}^{b} r_{i j}=\sum_{i=1}^{b} \frac{n_{i}}{n_{j}} r_{i j}=k \\
& \sum_{s=1}^{b} \frac{n_{s}}{n_{j}} r_{s i} r_{s j}=\delta_{i j}(k-\mu)+\mu n_{i}+(\lambda-\mu) r_{j i}
\end{aligned}
$$

where $0 \leq r_{i j} \leq n_{j}, 0 \leq r_{i i} \leq n_{i}-1$ and $\sum_{i=1}^{b} n_{i}=v$, is called $a$ row orbit matrix for a strongly regular graph with parameters $(v, k, \lambda, \mu)$ and the orbit lengths distribution $\left(n_{1}, \ldots, n_{b}\right)$.

Definition 3.2. $A(b \times b)$-matrix $C=\left[c_{i j}\right]$ with entries satisfying conditions:

$$
\begin{aligned}
& \sum_{i=1}^{b} c_{i j}=\sum_{j=1}^{b} \frac{n_{j}}{n_{i}} c_{i j}=k \\
& \sum_{s=1}^{b} \frac{n_{s}}{n_{j}} c_{i s} c_{j s}=\delta_{i j}(k-\mu)+\mu n_{i}+(\lambda-\mu) c_{i j}
\end{aligned}
$$

where $0 \leq c_{i j} \leq n_{i}, 0 \leq c_{i i} \leq n_{i}-1$ and $\sum_{i=1}^{b} n_{i}=v$, is called a column orbit matrix for a strongly regular graph with parameters $(v, k, \lambda, \mu)$ and the orbit lengths distribution $\left(n_{1}, \ldots, n_{b}\right)$.

The submatrix of an orbit matrix corresponding to the fixed vertices is called the fixed part of the orbit matrix, while the submatrix corresponding to the orbits of lengths greater than 1 is called the nonfixed part of the orbit matrix.

\section{Self orthogonal CODES From orbit MATrices of Strongly REGULAR GRAPHS}

In 2003 Harada and Tonchev introduced a method of constructing selforthogonal codes from orbit matrices of a design (see [12]). In [8] a method for constructing self-orthogonal codes from orbit matrices of strongly regular graphs admitting an automorphism group $G$ which acts with all orbits of the same length is described. These codes were defined over $F_{q}$, a finite field of prime order $q$, such that $q$ divides $k, \lambda, \mu$. Here we generalize that result to a construction of self-orthogonal codes from some submatrices of orbit matrices of strongly regular graphs, allowing the automorphism group to act in orbits that are not necessarily of the same length.

THEOREM 4.1. Let $\Gamma$ be a $\operatorname{SRG}(v, k, \lambda, \mu)$ having an automorphism group $G$ which acts on the set of vertices of $\Gamma$ with $b$ orbits of lengths $n_{1}, \ldots, n_{b}$, respectively, with $f$ fixed vertices, and the other $b-f$ orbits of lengths $n_{f+1}, \ldots, n_{b}$ divisible by $p$, where $p$ is a prime dividing $k, \lambda$ and $\mu$. Let 
$C$ be the column orbit matrix of the graph $\Gamma$ with respect to $G$. If $q$ is a prime power such that $q=p^{n}$, then the code spanned by the rows of the fixed part of the matrix $C$ is a self-orthogonal code of length $f$ over $F_{q}$.

Proof. From the definition of an orbit matrix, for $i^{\text {th }}$ and $j^{\text {th }}$ rows of $C$ we have

$$
\sum_{s=1}^{b} \frac{n_{s}}{n_{j}} c_{j s} c_{i s}=\delta_{i j}(k-\mu)+\mu n_{i}+(\lambda-\mu) c_{i j} .
$$

Let us assume that $n_{i}=n_{j}=1$. Then

$$
\sum_{s=1}^{b} \frac{n_{s}}{n_{j}} c_{j s} c_{i s}=\sum_{s=1}^{f} \frac{n_{s}}{n_{j}} c_{j s} c_{i s}+\sum_{s=f+1}^{b} \frac{n_{s}}{n_{j}} c_{j s} c_{i s}=\sum_{s=1}^{f} c_{j s} c_{i s}+\sum_{s=f+1}^{b} n_{s} c_{j s} c_{i s}
$$

where $p \mid n_{s}$ for $s=f+1, \ldots, b$. It follows that:

$$
\sum_{s=1}^{b} \frac{n_{s}}{n_{j}} c_{j s} c_{i s}-\sum_{s=f+1}^{b} n_{s} c_{j s} c_{i s}=\sum_{s=1}^{f} c_{j s} c_{i s}
$$

From (4.1) and (4.2), it follows that

$$
\begin{gathered}
\sum_{s=1}^{f} c_{j s} c_{i s}=\delta_{i j}(k-\mu)+\mu n_{i}+(\lambda-\mu) c_{i j}-\sum_{s=f+1}^{b} n_{s} c_{j s} c_{i s} \\
=\delta_{i j}(k-\mu)+\mu+(\lambda-\mu) c_{i j}-\sum_{s=f+1}^{b} n_{s} c_{j s} c_{i s} .
\end{gathered}
$$

If $i=j$, then

$$
\sum_{s=1}^{f} c_{j s} c_{i s}=k+(\lambda-\mu) c_{i j}-\sum_{s=f+1}^{b} n_{s} c_{j s} c_{i s}
$$

and if $i \neq j$, then

$$
\sum_{s=1}^{f} c_{j s} c_{i s}=\mu+(\lambda-\mu) c_{i j}-\sum_{s=f+1}^{b} n_{s} c_{j s} c_{i s}
$$

so the code spanned by the rows corresponding to the fixed part of the matrix $C$ is a self-orthogonal code of length $f$ over $F_{q}$.

In the following theorem we give a similar result for a submatrix of the nonfixed part of orbit matrices. 
THEOREM 4.2. Let $\Gamma$ be a $\operatorname{SRG}(v, k, \lambda, \mu)$ having an automorphism group $G$ which acts on the set of vertices of $\Gamma$ with $b$ orbits of lengths $n_{1}, \ldots, n_{b}$, respectively, such that there are $f$ fixed vertices, $h$ orbits of length $w$, and $b-f-h$ orbits of lengths $n_{f+h+1}, \ldots, n_{b}$. Further, let $p w \mid n_{s}$ if $w<n_{s}$, and $p n_{s} \mid w$ if $n_{s}<w$, for $s=f+h+1, \ldots, b$, where $p$ is a prime number dividing $k, \lambda, \mu$ and $w$. Let $C$ be the column orbit matrix of the graph $\Gamma$ with respect to $G$. If $q$ is a prime power such that $q=p^{n}$, then the code over $F_{q}$ spanned by the part of the matrix $C$ (rows and columns) determined by the orbits of length $w$ is a self-orthogonal code of length $h$.

Proof. Let $n_{1}=\ldots=n_{f}=1$ and $n_{f+1}=\ldots=n_{f+h}=w$. Further, let us assume that $n_{i}=n_{j}=w$. Then

$$
\begin{aligned}
\sum_{s=1}^{b} \frac{n_{s}}{n_{j}} c_{j s} c_{i s} & =\sum_{s=1}^{f} \frac{n_{s}}{n_{j}} c_{j s} c_{i s}+\sum_{s=f+1}^{f+h} \frac{n_{s}}{n_{j}} c_{j s} c_{i s}+\sum_{s=f+h+1}^{b} \frac{n_{s}}{n_{j}} c_{j s} c_{i s} \\
& =\sum_{s=1}^{f} \frac{1}{w} c_{j s} c_{i s}+\sum_{s=f+1}^{f+h} c_{j s} c_{i s}+\sum_{s=f+h+1}^{b} \frac{n_{s}}{w} c_{j s} c_{i s} .
\end{aligned}
$$

It follows that

$$
\sum_{s=1}^{b} \frac{n_{s}}{n_{j}} c_{j s} c_{i s}-\frac{1}{w} \sum_{s=1}^{f} c_{j s} c_{i s}-\sum_{s=f+h+1}^{b} \frac{n_{s}}{w} c_{j s} c_{i s}=\sum_{s=f+1}^{f+h} c_{j s} c_{i s} .
$$

If $s \in\{1,2, \ldots, f\}$, then $c_{j s}, c_{i s} \in\{0, w\}$, so

$$
c_{j s} c_{i s} \in\left\{0, w^{2}\right\} .
$$

From (4.1), (4.3) and (4.4) it follows that:

$$
\begin{aligned}
\sum_{s=f+1}^{f+h} c_{j s} c_{i s} & =\delta_{i j}(k-\mu)+\mu n_{i}+(\lambda-\mu) c_{i j}-\frac{f-x}{w} \cdot w^{2}-\sum_{s=f+h+1}^{b} \frac{n_{s}}{w} c_{j s} c_{i s} \\
& =\delta_{i j}(k-\mu)+\mu n_{i}+(\lambda-\mu) c_{i j}-w \cdot(f-x)-\sum_{s=f+h+1}^{b} \frac{n_{s}}{w} c_{j s} c_{i s}
\end{aligned}
$$

where $x=\left|\left\{s \in\{1, \ldots, f\}: c_{j s} c_{i s}=0\right\}\right|$. If $w<n_{s}$ then $p \mid \frac{n_{s}}{w}$. If $n_{s}<w$ then $p \mid \frac{n_{s}}{w} c_{j s} c_{i s}$, because $c_{i s}=r_{i s} \frac{n_{i}}{n_{s}}=r_{i s} \frac{w}{n_{s}}, c_{j s}=r_{j s} \frac{n_{j}}{n_{s}}=r_{j s} \frac{w}{n_{s}}$, and $p \mid \frac{w}{n_{s}}$. Hence $\sum_{s=f+1}^{f+h} c_{j s} c_{i s}$ is congruent to zero modulo $p$.

In Theorem 4.3 we give another construction of self-orthogonal codes from orbit matrices of strongly regular graphs. 
THEOREM 4.3. Let $\Gamma$ be a $\operatorname{SRG}(v, k, \lambda, \mu)$ with an automorphism group $G$ which acts on the set of vertices of $\Gamma$ with $b$ orbits of lengths $n_{1}, \ldots, n_{b}$, respectively, and $w=\max \left\{n_{1}, \ldots, n_{b}\right\}$. Further, let $p$ be a prime dividing $k$, $\lambda, \mu$ and $w$, and let $p n_{s} \mid w$ if $n_{s} \neq w$. Let $C$ be the column orbit matrix of the graph $\Gamma$ with respect to $G$. If $q$ is a prime power such that $q=p^{n}$, then the code over $F_{q}$ spanned by the rows of $C$ corresponding to the orbits of length $w$ is a self-orthogonal code of length $b$.

Proof. Let $n_{i}=n_{j}=w$. We have

$$
\sum_{s=1}^{b} c_{j s} c_{i s}=\sum_{s, n_{s}<w} c_{j s} c_{i s}+\sum_{s, n_{s}=w} c_{j s} c_{i s} .
$$

Since $c_{i s}=r_{i s} \frac{n_{i}}{n_{s}}=r_{i s} \frac{w}{n_{s}}$ and $c_{j s}=r_{j s} \frac{n_{j}}{n_{s}}=r_{j s} \frac{w}{n_{s}}$, it follows that $c_{j s} c_{i s}$ is divisible by $p$ if $n_{s}<w$. Further, by Theorem $4.2, \sum_{s, n_{s}=w} c_{j s} c_{i s}$ is divisible by $p$. Hence, we conclude that $\sum_{s=1}^{b} c_{j s} c_{i s}$ is congruent to zero modulo $p$.

THEOREM 4.4. Let $\Gamma$ be a $\operatorname{SRG}(v, k, \lambda, \mu)$ with an automorphism group $G$ which acts on the set of vertices of $\Gamma$ with $b$ orbits of lengths $n_{1}, \ldots, n_{b}$, respectively, and $w=\min \left\{n_{1}, \ldots, n_{b}\right\}$. Further, let $p$ be a prime dividing $k$, $\lambda, \mu$ and $w$, and let $p w \mid n_{s}$ if $n_{s} \neq w$. Let $R$ be the row orbit matrix of the graph $\Gamma$ with respect to $G$. If $q$ is a prime power such that $q=p^{n}$, then the code over $F_{q}$ spanned by the rows of $R$ corresponding to the orbits of length $w$ is a self-orthogonal code of length $b$.

Proof. Let $n_{i}=n_{j}=w$. We have

$$
\sum_{s=1}^{b} r_{j s} r_{i s}=\sum_{s, n_{s}=w} r_{j s} r_{i s}+\sum_{s, n_{s}>w} r_{j s} r_{i s}
$$

Since $r_{i s}=c_{i s} \frac{n_{s}}{w}$ and $r_{j s}=c_{j s} \frac{n_{s}}{w}$, it follows that $r_{j s} r_{i s}$ is divisible by $p$ if $n_{s}>w$.

We have that

$$
\sum_{s, n_{s}=w} r_{j s} r_{i s}=\sum_{s, n_{s}=w} c_{j s} \frac{n_{s}}{n_{j}} c_{i s} \frac{n_{s}}{n_{i}}=\sum_{s, n_{s}=w} c_{j s} c_{i s}
$$

and by Theorem $4.2, \sum_{s, n_{s}=w} c_{j s} c_{i s}$ is divisible by $p$.

Hence, we conclude that $\sum_{s=1}^{b} r_{j s} r_{i s}$ is congruent to zero modulo $p$.

\section{BINARY LINEAR CODES FROM ORBIT MATRICES OF TRIANGULAR GRAPHS ON UP TO 120 VERTICES}

Codes spanned by adjacency matrices of triangular graphs have been extensively studied, for example by Tonchev [16, p. 171], Haemers et al. [11, Theorem 4.1] and Key et al. [14]. Results on codes from adjacency matrices of triangular graphs can also be found in $[1,5,6,9]$. 
In this section we construct self-orthogonal codes from orbit matrices of triangular graphs by applying theorems presented in Section 4. In order to apply Theorems 4.1, 4.2, 4.3 and 4.4, we take into consideration triangular graphs $T(2 k)$, having the parameter $\lambda=2 k-2$ even. First we construct orbit matrices for $Z_{2}$ acting on triangular graphs $T(2 k)$, where $3 \leq k \leq 8$. For the construction of orbit matrices we were using programs written for $\operatorname{GAP}([10])$. Then we apply Theorem 4.1 and Theorem 4.2 to construct binary self-orthogonal codes from the orbit matrices. After that we consider orbit matrices with respect to $Z_{4}$. The codes were analyzed using Magma [4].

5.1. Codes from orbit matrices with respect to $Z_{2}$. In order to construct the orbit matrices of involutions action on the triangular graphs $T(n)$, for $n \in$ $\{6,8,10,12,14,16\}$, we have to determine the conjugacy classes of subgroups of order two in the full automorphism groups of the triangular graphs. It is known that the full automorphism group of the triangular graph $T(n)$ is isomorphic to $S_{n}$. The information on the subgroups of $S_{n}$ of order two and the number of their fixed points is given in Table 1 . In Tables 2 and 3 we present information on the obtained codes, omitting the trivial codes.

TABLE 1. Orbits of subgroups of $\operatorname{Aut}(T(n))$, up to conjugation, isomorphic to $Z_{2}$

\begin{tabular}{|c|c|c||c|c|c|}
\hline$T(n)$ & \# fixed points & \# subgroups & $T(n)$ & \# fixed points & \# subgroups \\
\hline \hline$T(6)$ & 3 & 2 & $T(14)$ & 7 & 2 \\
\hline$T(6)$ & 7 & 1 & $T(14)$ & 11 & 1 \\
\hline$T(8)$ & 4 & 2 & $T(14)$ & 19 & 1 \\
\hline$T(8)$ & 8 & 1 & $T(14)$ & 31 & 1 \\
\hline$T(8)$ & 16 & 1 & $T(14)$ & 47 & 1 \\
\hline$T(10)$ & 5 & 2 & $T(14)$ & 67 & 1 \\
\hline$T(10)$ & 9 & 1 & $T(16)$ & 8 & 2 \\
\hline$T(10)$ & 17 & 1 & $T(16)$ & 12 & 1 \\
\hline$T(10)$ & 29 & 1 & $T(16)$ & 20 & 1 \\
\hline$T(12)$ & 6 & 2 & $T(16)$ & 32 & 1 \\
\hline$T(12)$ & 10 & 1 & $T(16)$ & 48 & 1 \\
\hline$T(12)$ & 18 & 1 & $T(16)$ & 68 & 1 \\
\hline$T(12)$ & 30 & 1 & $T(16)$ & 92 & 1 \\
\hline$T(12)$ & 46 & 1 & & & \\
\hline
\end{tabular}

Some of the constructed codes are optimal or near-optimal. Namely, the codes with parameters $[6,2,4],[7,2,4],[10,2,6],[12,2,8],[12,3,6],[16,4,8]$, $[17,4,8],[18,4,8]$ and $[30,4,16]$ are optimal, and near-optimal codes are the ones with parameters $[8,2,4],[14,2,8],[19,4,8]$ and $[29,6,12]$.

5.2. Codes from orbit matrices with respect to $Z_{4}$. A group isomorphic to $Z_{4}$ can act with orbits of lengths 1, 2 and 4 . In Table 4 we give information on orbit lengths distributions of subgroups of $\operatorname{Aut}(T(n))$ isomorphic to $Z_{4}$ and 
TABLE 2. Codes from the fixed parts of orbit matrices for $Z_{2}$ acting on $T(2 k), 3 \leq k \leq 8$

\begin{tabular}{|c|c|c|c|}
\hline$T(2 k)$ & $C$ & $\mid$ Aut $(C) \mid$ & Weight Distribution \\
\hline \hline $3 \leq k \leq 8$ & {$[k+4,2,4]$} & $2 \cdot 4 !(k-2) !$ & {$[<0,1>,<4,3>]$} \\
\hline $4 \leq k \leq 8$ & {$[k+12,4,8]$} & $4 \cdot 7 !(k-3) !$ & {$[<0,1>,<8,15>]$} \\
\hline $5 \leq k \leq 8$ & {$[k+24,6,12]$} & $8 !(k-4) !$ & {$[<0,1>,<12,28>,<16,35>]$} \\
\hline $6 \leq k \leq 8$ & {$[k+40,8,16]$} & $10 !(k-5) !$ & {$[<0,1>,<16,45>,<24,210>]$} \\
\hline $7 \leq k \leq 8$ & {$[k+60,10,20]$} & $12 !(k-6) !$ & {$[<0,1>,<20,66>,<32,495>,<36,462>]$} \\
\hline$k=8$ & {$[k+84,12,24]$} & $14 !(k-7) !$ & {$[<0,1>,<24,91>,<40,1001>,<48,3003>]$} \\
\hline
\end{tabular}

TABLE 3. Codes from the nonfixed parts of orbit matrices for $Z_{2}$ acting on $T(2 k), 3 \leq k \leq 8$

\begin{tabular}{|c|l|l|l|}
\hline$T(n)$ & \multicolumn{1}{|c|}{$C$} & $\mid$ Aut $(C) \mid$ & WeightDistribution \\
\hline$T(6)$ & {$[6,2,4]$} & $2^{4} 3$ & {$[<0,1>,<4,3>]$} \\
\hline$T(8)$ & {$[10,2,6]$} & $2^{8} 3^{2}$ & {$[<0,1>,<6,2>,<8,1>]$} \\
\hline$T(8)$ & {$[12,2,8]$} & $2^{10} 3^{4}$ & {$[<0,1>,<8,3>]$} \\
\hline$T(8)$ & {$[12,3,6]$} & $2^{9} 3$ & {$[<0,1>,<6,4>,<8,3>]$} \\
\hline$T(10)$ & {$[14,2,8]$} & $2^{10} 3^{4} 5^{2}$ & {$[<0,1>,<8,2>,<12,1>]$} \\
\hline$T(10)$ & {$[18,3,8]$} & $2^{13} 3^{4}$ & {$[<0,1>,<8,3>,<12,4>]$} \\
\hline$T(10)$ & {$[20,4,8]$} & $2^{13} 3^{1} 5^{1}$ & {$[<0,1>,<8,5>,<12,10>]$} \\
\hline$T(12)$ & {$[18,2,10]$} & $2^{16} 3^{4} 5^{2} 7^{2}$ & {$[<0,1>,<10,2>,<16,1>]$} \\
\hline$T(12)$ & {$[24,3,10]$} & $2^{16} 3^{7} 5^{3}$ & {$[<0,1>,<10,3>,<16,3>,<18,1>]$} \\
\hline$T(12)$ & {$[28,4,10]$} & $2^{21} 3^{5}$ & {$[<0,1>,<10,4>,<16,7>,<18,4>]$} \\
\hline$T(12)$ & {$[30,5,10]$} & $2^{19} 3^{2} 5^{1}$ & {$[<0,1>,<10,6>,<16,15>,<18,10>]$} \\
\hline$T(12)$ & {$[30,4,16]$} & $2^{21} 3^{2} 5^{1} 7^{1}$ & {$[<0,1>,<16,15>]$} \\
\hline$T(14)$ & {$[22,2,12]$} & $2^{18} 3^{8} 5^{4} 7^{2}$ & {$[<0,1>,<12,2>,<20,1>]$} \\
\hline$T(14)$ & {$[30,3,12]$} & $2^{25} 3^{7} 5^{3} 7^{3}$ & {$[<0,1>,<12,3>,<20,3>,<24,1>]$} \\
\hline$T(14)$ & {$[36,4,12]$} & $2^{25} 3^{9} 5^{4}$ & {$[<0,1>,<12,4>,<20,6>,<24,5>]$} \\
\hline$T(14)$ & {$[40,5,12]$} & $2^{28} 3^{6} 5^{1}$ & {$[<0,1>,<12,5>,<20,11>,<24,15>]$} \\
\hline$T(14)$ & {$[42,6,12]$} & $2^{25} 3^{2} 5^{1} 7^{1}$ & {$[<0,1>,<12,7>,<20,21>,<24,35>]$} \\
\hline$T(16)$ & {$[26,2,14]$} & $2^{22} 3^{10} 5^{4} 7^{2} 11^{2}$ & {$[<0,1>,<14,2>,<24,1>]$} \\
\hline$T(16)$ & {$[36,3,14]$} & $2^{28} 3^{13} 5^{6} 7^{3}$ & {$[<0,1>,<14,3>,<24,3>,<30,1>]$} \\
\hline$T(16)$ & {$[44,4,14]$} & $2^{37} 3^{9} 5^{4} 7^{4}$ & {$[<0,1>,<14,4>,<24,6>,<30,4>,<32,1>]$} \\
\hline$T(16)$ & {$[50,5,14]$} & $2^{33} 3^{11} 5^{6}$ & {$[<0,1>,<14,5>,<24,10>,<30,11>,<32,5>]$} \\
\hline$T(16)$ & {$[54,6,14]$} & $2^{37} 3^{8} 5^{1}$ & {$[<0,1>,<14,6>,<24,16>,<30,26>,<32,15>]$} \\
\hline$T(16)$ & {$[56,6,24]$} & $2^{35} 3^{2} 5^{1} 7^{1}$ & {$[<0,1>,<24,28>,<32,35>]$} \\
\hline$T(16)$ & {$[56,7,14]$} & $2^{35} 3^{2} 5^{1} 7^{1}$ & {$[<0,1>,<14,8>,<24,28>,<30,56>,<32,35>]$} \\
\hline
\end{tabular}

their orbits on $T(n)$, for $n \in\{6,8,10,12,14,16\}$. Then we present information on binary codes spanned by parts of orbit matrices, using results given in Section 4. The codes obtained using Theorem 4.1 are presented in Table 5, and the codes obtained applying Theorem 4.2 are given in Tables 6 and 7 .

The codes with parameters $[6,2,4],[7,2,4],[13,2,8],[15,4,8],[16,4,8]$, $[17,4,8],[18,4,8],[28,6,12]$ and $[31,4,16]$ are optimal, and the codes with parameters $[8,2,4],[11,2,6],[13,3,6],[14,2,8]$ and $[29,6,12]$ are near-optimal.

The information on codes constructed from column orbit matrices for $Z_{4}$ by applying Theorem 4.3 are given in Table 8 . In that case binary codes are spanned by the rows of orbit matrices that correspond to orbits of length 
TABLE 4. Orbits of subgroups of $\operatorname{Aut}(T(n))$, up to conjugation, isomorphic to $Z_{4}$

\begin{tabular}{|c|c|c|c|c|}
\hline$T(n)$ & \# subgroups & $\begin{array}{l}\text { \# fixed } \\
\text { points }\end{array}$ & $\begin{array}{c}\text { \# orbits of } \\
\text { length } 2\end{array}$ & $\begin{array}{c}\text { \# orbits of } \\
\text { length } 4\end{array}$ \\
\hline$T(6)$ & 2 & 1 & 1 & 3 \\
\hline$T(8)$ & 1 & 0 & 2 & 6 \\
\hline$T(8)$ & 2 & 2 & 3 & 5 \\
\hline$T(8)$ & 1 & 6 & 1 & 5 \\
\hline$T(10)$ & 2 & 1 & 2 & 10 \\
\hline$T(10)$ & 2 & 3 & 7 & 7 \\
\hline$T(10)$ & 1 & 7 & 5 & 7 \\
\hline$T(10)$ & 1 & 15 & 1 & 7 \\
\hline$T(12)$ & 1 & 0 & 3 & 15 \\
\hline$T(12)$ & 2 & 2 & 4 & 14 \\
\hline$T(12)$ & 2 & 4 & 13 & 9 \\
\hline$T(12)$ & 1 & 6 & 2 & 14 \\
\hline$T(12)$ & 1 & 8 & 11 & 9 \\
\hline$T(12)$ & 1 & 16 & 7 & 9 \\
\hline$T(12)$ & 1 & 28 & 1 & 9 \\
\hline$T(14)$ & 2 & 1 & 3 & 21 \\
\hline$T(14)$ & 2 & 3 & 8 & 18 \\
\hline$T(14)$ & 2 & 5 & 21 & 11 \\
\hline$T(14)$ & 1 & 7 & 6 & 18 \\
\hline$T(14)$ & 1 & 9 & 19 & 11 \\
\hline$T(14)$ & 1 & 15 & 2 & 18 \\
\hline$T(14)$ & 1 & 17 & 15 & 11 \\
\hline$T(14)$ & 1 & 29 & 9 & 11 \\
\hline$T(14)$ & 1 & 45 & 1 & 11 \\
\hline$T(16)$ & 1 & 0 & 4 & 28 \\
\hline$T(16)$ & 2 & 2 & 5 & 27 \\
\hline$T(16)$ & 2 & 4 & 14 & 22 \\
\hline$T(16)$ & 2 & 6 & 31 & 13 \\
\hline$T(16)$ & 1 & 6 & 3 & 27 \\
\hline$T(16)$ & 1 & 8 & 12 & 22 \\
\hline$T(16)$ & 1 & 10 & 29 & 13 \\
\hline$T(16)$ & 1 & 16 & 8 & 22 \\
\hline$T(16)$ & 1 & 18 & 25 & 13 \\
\hline$T(16)$ & 1 & 28 & 2 & 22 \\
\hline$T(16)$ & 1 & 30 & 19 & 13 \\
\hline$T(16)$ & 1 & 46 & 11 & 13 \\
\hline$T(16)$ & 1 & 66 & 1 & 13 \\
\hline
\end{tabular}

4. The codes obtained from parts of row orbit matrices for $Z_{4}$ acting without fixed vertices corresponding to the orbits of length 4, constructed using Theorem 4.4, have dimension zero. 
TABLE 5. Codes from the fixed part of orbit matrices for $Z_{4}$ acting on $T(2 k), 3 \leq k \leq 8$

\begin{tabular}{|c|c|c|c|}
\hline$T(2 k)$ & $C$ & $|\operatorname{Aut}(C)|$ & Weight Distribution \\
\hline$k=4,6,8$ & {$[6,2,4]$} & $2^{4} 3^{1}$ & $[<0,1\rangle,<4,3\rangle]$ \\
\hline$k=5,7$ & {$[7,2,4]$} & $2^{4} 3^{1}$ & $[<0,1\rangle,<4,3\rangle]$ \\
\hline$k=6,8$ & {$[8,2,4]$} & $2^{5} 3^{1}$ & $[<0,1\rangle,<4,3\rangle]$ \\
\hline$k=7,8$ & {$[\mathrm{k}+2,2,4]$} & $2^{2 k-9} 3^{2}$ & $[<0,1\rangle,\langle 4,3\rangle]$ \\
\hline$k=5,7$ & {$[15,4,8]$} & $2^{6} 3^{2} 5^{1} 7^{1}$ & {$[<0,1>,<8,15>]$} \\
\hline$k=6,8$ & {$[16,4,8]$} & $2^{6} 3^{2} 5^{1} 7^{1}$ & {$[<0,1>,<8,15>]$} \\
\hline$k=7,8$ & {$[\mathrm{k}+10,4,8]$} & $2^{7} 3^{k-5} 5^{1} 7^{1}$ & $[<0,1\rangle,<8,15\rangle]$ \\
\hline$k=6,8$ & {$[28,6,12]$} & $2^{7} 3^{2} 5^{1} 7^{1}$ & $[<0,1\rangle,<12,28\rangle,<16,35\rangle]$ \\
\hline$k=7,8$ & {$[\mathrm{k}+22,6,12]$} & $2^{k} 3^{2} 5^{1} 7^{1}$ & {$[<0,1>,<12,28>,<16,35>]$} \\
\hline$k=7,8$ & {$[\mathrm{k}+38,8,16]$} & $2^{8} 3^{4} 5^{2} 7^{1}$ & $[<0,1\rangle,\langle 16,45\rangle,<24,210\rangle]$ \\
\hline$k=8$ & {$[66,10,20]$} & $2^{10} 3^{5} 5^{2} 7^{1} 11^{1}$ & {$[<0,1>,<20,66>,<32,495>,<36,462>$} \\
\hline
\end{tabular}

TABLE 6 . Codes from parts of orbit matrices for $Z_{4}$ corresponding to the orbits of length 2

\begin{tabular}{|c|c|l|l|}
\hline$T(n)$ & \multicolumn{1}{|c|}{$C$} & $\mid$ Aut $(C) \mid$ & Weight Distribution \\
\hline$T(10)$ & {$[7,2,4]$} & $2^{4} 3^{1}$ & {$[<0,1>,<4,3>]$} \\
\hline$T(12)$ & {$[11,2,6]$} & $2^{8} 3^{2}$ & {$[<0,1>,<6,2>,<8,1>]$} \\
\hline$T(12)$ & {$[13,2,8]$} & $2^{10} 3^{4}$ & {$[<0,1>,<8,3>]$} \\
\hline$T(12)$ & {$[13,3,6]$} & $2^{9} 3^{1}$ & {$[<0,1>,<6,4>,<8,3>]$} \\
\hline$T(14)$ & {$[8,2,4]$} & $2^{5} 3^{1}$ & {$[<0,1>,<4,3>]$} \\
\hline$T(14)$ & {$[15,2,8]$} & $2^{10} 3^{4} 5^{2}$ & {$[<0,1>,<8,2>,<12,1>]$} \\
\hline$T(14)$ & {$[19,3,8]$} & $2^{13} 3^{4}$ & {$[<0,1>,<8,3>,<12,4>]$} \\
\hline$T(14)$ & {$[21,4,8]$} & $2^{13} 3^{1} 5^{1}$ & {$[<0,1>,<8,5>,<12,10>]$} \\
\hline$T(16)$ & {$[12,2,6]$} & $2^{9} 3^{2}$ & {$[<0,1>,<6,2>,<8,1>]$} \\
\hline$T(16)$ & {$[14,2,8]$} & $2^{11} 3^{4}$ & {$[<0,1>,<8,3>]$} \\
\hline$T(16)$ & {$[14,3,6]$} & $2^{10} 3^{1}$ & {$[<0,1>,<6,4>,<8,3>]$} \\
\hline$T(16)$ & {$[19,2,10]$} & $2^{16} 3^{4} 5^{2} 7^{2}$ & {$[<0,1>,<10,2>,<16,1>]$} \\
\hline$T(16)$ & {$[25,3,10]$} & $2^{16} 3^{7} 5^{3}$ & {$[<0,1>,<10,3>,<16,3>,<18,1>]$} \\
\hline$T(16)$ & {$[29,4,10]$} & $2^{21} 3^{5}$ & {$[<0,1>,<10,4>,<16,7>,<18,4>]$} \\
\hline$T(16)$ & {$[31,4,16]$} & $2^{21} 3^{2} 5^{1} 7^{1}$ & {$[<0,1>,<16,15>]$} \\
\hline$T(16)$ & {$[31,5,10]$} & $2^{19} 3^{2} 5^{1}$ & {$[<0,1>,<10,6>,<16,15>,<18,10>]$} \\
\hline
\end{tabular}

TABLE 7. Codes from parts of orbit matrices for $Z_{4}$ corresponding to the orbits of length 4

\begin{tabular}{|c|c|l|l|}
\hline$T(n)$ & \multicolumn{1}{|c|}{$C$} & $\mid$ Aut $\left(C_{C_{i}}\right) \mid$ & Weight Distribution \\
\hline$T(10)$ & {$[10,2,4]$} & $2^{7} 3^{1}$ & {$[<0,1>,<4,1>,<6,2>]$} \\
\hline$T(12)$ & {$[14,2,8]$} & $2^{11} 3^{4}$ & {$[<0,1>,<8,3>]$} \\
\hline$T(12)$ & {$[15,2,8]$} & $2^{11} 3^{5}$ & {$[<0,1>,<8,3>]$} \\
\hline$T(14)$ & {$[18,2,10]$} & $2^{13} 3^{5} 5^{2}$ & {$[<0,1>,<10,2>,<12,1>]$} \\
\hline$T(14)$ & {$[21,3,6]$} & $2^{14} 3^{5}$ & {$[<0,1>,<6,1>,<10,3>,<12,3>]$} \\
\hline$T(16)$ & {$[22,2,12]$} & $2^{19} 3^{5} 5^{2} 7^{2}$ & {$[<0,1>,<12,2>,<16,1>]$} \\
\hline$T(16)$ & {$[27,3,12]$} & $2^{22} 3^{8}$ & {$[<0,1>,<12,4>,<16,3>]$} \\
\hline$T(16)$ & {$[28,2,16]$} & $2^{25} 3^{8} 5^{3} 7^{3}$ & {$[<0,1>,<16,3>]$} \\
\hline
\end{tabular}


TABLE 8. Codes from orbit matrices for $Z_{4}$, applying Theorem 4.3

\begin{tabular}{|l|l|l|l|}
\hline$T(n)$ & \multicolumn{1}{|c|}{$C$} & $\mid$ Aut $(C) \mid$ & WeightDistribution \\
\hline$T(10)$ & {$[13,2,6]$} & $2^{9} 3^{2} 5^{1}$ & {$[<0,1>,<4,1>,<6,2>]$} \\
\hline$T(12)$ & {$[18,2,8]$} & $2^{14} 3^{6} 5^{1}$ & {$[<0,1>,<8,3>]$} \\
\hline$T(12)$ & {$[20,2,8]$} & $2^{17} 3^{6} 5^{1} 7^{1}$ & {$[<0,1>,<8,3>]$} \\
\hline$T(12)$ & {$[22,2,8]$} & $2^{18} 3^{8} 5^{2} 7^{1}$ & {$[<0,1>,<8,3>]$} \\
\hline$T(14)$ & {$[25,3,6]$} & $2^{17} 3^{6} 5^{1} 7^{1}$ & {$[<0,1>,<6,1>,<10,3>,<12,3>]$} \\
\hline$T(14)$ & {$[29,2,10]$} & $2^{22} 3^{10} 5^{4} 7^{1} 11^{1} 13^{1}$ & {$[<0,1>,<10,2>,<12,1>]$} \\
\hline$T(14)$ & {$[31,2,10]$} & $2^{23} 3^{11} 5^{5} 7^{2} 11^{1} 13^{1}$ & {$[<0,1>,<10,2>,<12,1>]$} \\
\hline$T(14)$ & {$[35,2,10]$} & $2^{28} 3^{13} 5^{5} 7^{2} 11^{1} 13^{1} 17^{1} 19^{1}$ & {$[<0,1>,<10,2>,<12,1>]$} \\
\hline$T(16)$ & {$[32,2,16]$} & $2^{29} 3^{9} 5^{4} 7^{4}$ & {$[<0,1>,<16,3>]$} \\
\hline$T(16)$ & {$[34,3,12]$} & $2^{29} 3^{11} 5^{2} 7^{1}$ & {$[<0,1>,<12,4>,<16,3>]$} \\
\hline$T(16)$ & {$[36,3,12]$} & $2^{31} 3^{12} 5^{2} 7^{1} 11^{1}$ & {$[<0,1>,<12,4>,<16,3>]$} \\
\hline$T(16)$ & {$[40,2,12]$} & $2^{36} 3^{13} 5^{6} 7^{4} 11^{1} 13^{1} 17^{1} 19^{1}$ & {$[<0,1>,<12,2>,<16,1>]$} \\
\hline$T(16)$ & {$[42,2,12]$} & $2^{37} 3^{14} 5^{6} 7^{5} 11^{2} 13^{1} 17^{1} 19^{1}$ & {$[<0,1>,<12,2>,<16,1>]$} \\
\hline$T(16)$ & {$[46,2,12]$} & $2^{41} 3^{15} 5^{8} 7^{5} 11^{2} 13^{2} 17^{1} 19^{1} 23^{1}$ & {$[<0,1>,<12,2>,<16,1>]$} \\
\hline$T(16)$ & {$[52,2,12]$} & $2^{49} 3^{19} 5^{9} 7^{6} 11^{2} 13^{2} 17^{1} 19^{1} 23^{1} 29^{1} 31^{1}$ & {$[<0,1>,<12,2>,<16,1>]$} \\
\hline
\end{tabular}

\section{SRGs AND BIBDs CONSTRUCTED FROM CODES}

In this section we use the codes constructed in Section 5 to obtain strongly regular graphs and block designs. In order to construct strongly regular graphs we consider a set of codewords of certain weight $w$ and look at the pairwise distances of the codewords. We identify the vertices of the graph by the codewords of weight $w$ and define adjacency with respect to the Hamming distance of the codewords. In some cases the constructed graphs are strongly regular. We observe the cases when the distances between two codewords take two, three or four values.

If there are two possible values for a distance between two codewords, denoted by $d_{1}$ and $d_{2}$, then we define two vertices $x$ and $y$ to be adjacent if and only if $d(x, y)=d_{1}$ (for the complementary graph we define that $x$ and $y$ are adjacent if and only if $\left.d(x, y)=d_{2}\right)$.

If there are three possible values for a distance between two codewords, namely $d_{1}$ and $d_{2}$ and $d_{3}$, we have more possibilities to define adjacency. Firstly, we define two vertices $x$ and $y$ to be adjacent if and only if $d(x, y)=d_{1}$, Secondly, two vertices $x$ and $y$ are adjacent if and only if $d(x, y)=d_{2}$, and thirdly, two vertices $x$ and $y$ are adjacent if and only if $d(x, y)=d_{3}$.

Let there be four values for a distance between two codewords, $d_{1}$ and $d_{2}$, $d_{3}$ and $d_{4}$. Firstly, we define two vertices $x$ and $y$ to be adjacent if and only if $d(x, y)=d_{1}$, or $d(x, y)=d_{2}$, secondly, two vertices $x$ and $y$ are adjacent if and only if $d(x, y)=d_{1}$ or $d(x, y)=d_{3}$ and thirdly, two vertices $x$ and $y$ are adjacent if and only if $d(x, y)=d_{1}$ or $d(x, y)=d_{4}$. Further, we define adjacency taking into cosideration only one intersection $\left(d_{1}, d_{2}, d_{3}\right.$ or $\left.d_{4}\right)$.

The construction is conducted using the GAP package Grape [15]. The obtained strongly regular graphs are presented in Tables 9, 10, 11, 12, 13, 14 and 15 . 
TABLE 9. SRGs from codes spanned by fixed parts of orbit matrices for $Z_{2}$, the case with two intersections of codewords

\begin{tabular}{|c|c|c|}
\hline$(v, k, \lambda, \mu)$ & $\mid$ Aut $(G) \mid$ & From triangular graphs $T(2 k)$ \\
\hline \hline$(28,12,6,4)$ & $8 !$ & $5 \leq k \leq 8$ \\
$(35,16,6,8)$ & $8 !$ & $5 \leq k \leq 8$ \\
$(45,16,8,4)$ & $10 !$ & $6 \leq k \leq 8$ \\
$(66,20,10,4)$ & $12 !$ & $7 \leq k \leq 8$ \\
$(91,24,12,4)$ & $14 !$ & $k=8$ \\
\hline
\end{tabular}

The constructed strongly regular graphs with parameters $(28,12,6,4)$, $(45,16,8,4),(66,20,10,4)$ and $(91,24,12,4)$ are the triangular graphs $T(8)$, $T(10), T(12)$ and $T(14)$, respectively. The constructed strongly regular graph with parameters $(35,16,6,8)$ is the complement of the distance 2 graph in the Johnson graph $J(7,4)$. The full automorphism group of that $\operatorname{SRG}(35,16,6,8)$ is isomorphic to $S_{8}$.

TABLE 10. SRGs from codes spanned by fixed parts of orbit matrices for $Z_{2}$, the case with three intersections of codewords

\begin{tabular}{|c|c|c|}
\hline$(v, k, \lambda, \mu)$ & $|\operatorname{Aut}(G)|$ & From triangular graphs $T(2 k)$ \\
\hline \hline$(495,238,109,119)$ & $2^{21} 3^{6} 5^{2} 7 \cdot 11 \cdot 17$ & $7 \leq k \leq 8$ \\
\hline
\end{tabular}

The constructed SRG(495,238,109,119) is the distance 2 or 4 graph in the Johnson graph $J(12,4)$. Its full automorphism group is isomorphic to the group $O_{10}^{-}(2): Z_{2}$.

TABLE 11. SRGs from codes spanned by nonfixed parts of orbit matrices for $Z_{2}$, the case with two intersections of codewords

\begin{tabular}{|c|c|c|}
\hline$(v, k, \lambda, \mu)$ & $\mid$ Aut $(G) \mid$ & From triangular graphs $T(2 k)$ \\
\hline \hline$(10,3,0,1)$ & $5 !$ & $k=5,8$ \\
$(15,8,4,4)$ & $6 !$ & $7 \leq k \leq 8$ \\
$(21,10,5,4)$ & $7 !$ & $k=7$ \\
$(28,12,6,4)$ & $8 !$ & $k=8$ \\
$(35,16,6,8)$ & $8 !$ & $k=8$ \\
\hline
\end{tabular}

The strongly regular graph with parameters $(10,3,0,1)$ is the Petersen graph, the unique SRG with these parameters. The constructed strongly regular graphs with parameters $(15,8,4,4),(21,10,5,4)$ and $(28,12,6,4)$ are the triangular graphs $T(6), T(7)$ and $T(8)$, respectively. The $\operatorname{SRG}(35,16,6,8)$ is isomorphic to SRG with the same parameters constructed from the fixed parts of orbit matrices. 
TABLE 12. SRGs from codes spanned by nonfixed parts of orbit matrices for $Z_{2}$, the case with three intersections of codewords

\begin{tabular}{|c|c|c|}
\hline$(v, k, \lambda, \mu)$ & $\mid$ Aut $(G) \mid$ & From triangular graphs $T(2 k)$ \\
\hline \hline$(15,8,4,4)$ & $6 !$ & $k=7$ \\
$(35,16,6,8)$ & $8 !$ & $k=7$ \\
\hline
\end{tabular}

The strongly regular graphs with parameters $(15,8,4,4)$ and $(35,16,6,8)$ obtained from codes spanned by nonfixed parts of orbit matrices for $Z_{2}$, in the case with three possible intersections of codewords, are isomorphic to the graphs with the same parameters obtained in the case with two intersections of codewords.

In Tables 13, 14 and 15 we list SRGs constructed from codes spanned by the fixed parts of orbit matrices for $Z_{4}$. In cases when there are two possible intersections of codewords the obtained SRGs are triangular graphs, the complement of the distance 2 graph in the Johnson graph $J(7,4)$, or the Petersen graph. Inspecting the case when there are three possible intersections of codewords we obtained a $\operatorname{SRG}(495,238,109,119)$ which is the distance 2 or 4 graph in the Johnson graph $J(12,4)$, i.e. the same graph as we obtained from orbit matrices with respect to $Z_{2}$.

TABLE 13. SRGs from codes spanned by fixed parts of orbit matrices for $Z_{4}$, the case with two intersections of codewords

\begin{tabular}{|c|c|c|}
\hline$(v, k, \lambda, \mu)$ & $\mid$ Aut $(G) \mid$ & From triangular graphs $T(2 k)$ \\
\hline$(28,12,6,4)$ & $8 !$ & $6 \leq k \leq 8$ \\
\hline$(35,16,6,8)$ & $8 !$ & $6 \leq k \leq 8$ \\
\hline$(45,16,8,4)$ & $10 !$ & $7 \leq k \leq 8$ \\
\hline$(66,20,10,4)$ & $12 !$ & $k=8$ \\
\hline
\end{tabular}

TABLE 14. SRGs from codes spanned by fixed parts of orbit matrices for $Z_{4}$, the case with three intersections of codewords

\begin{tabular}{|c|c|c|}
\hline$(v, k, \lambda, \mu)$ & $|\operatorname{Aut}(G)|$ & From triangular graphs $T(2 k)$ \\
\hline \hline$(495,238,109,119)$ & $2^{21} 3^{6} 5^{2} 7 \cdot 11 \cdot 17$ & $k=8$ \\
\hline
\end{tabular}

TABLE 15. SRGs from codes spanned by parts of orbit matrices for $Z_{4}$ corresponding to orbits of length 2 , the case with two intersections of codewords

\begin{tabular}{|c|c|c|}
\hline$(v, k, \lambda, \mu)$ & $|\operatorname{Aut}(G)|$ & From triangular graphs $T(2 k)$ \\
\hline \hline$(10,3,0,1)$ & $5 !$ & $k=7$ \\
\hline
\end{tabular}


We also use the codes from Section 5 to construct BIBDs, taking into consideration a set of codewords of certain weight. Identifying coordinate positions of the codes with the points, and codewords of a weight $k$ with blocks, we obtain an incidence structure having all blocks of size $k$. In other words, we consider the support designs of the constructed codes. In some cases these support designs are BIBDs. In Tables 16 and 17 we present information on the obtained BIBDs. Note that we do not list symmetric designs constructed from strongly regular graphs having $\lambda=\mu$. The support designs obtained from the codes of nonfixed parts of orbit matrices for $Z_{2}$ acting on $T(12)$ are BIBDs with repeated blocks. In that case we also give parameters of the corresponding simple designs.

TABLE 16. BIBDs from the codes of nonfixed parts of orbit matrices for $Z_{2}$ acting on $T(12)$

\begin{tabular}{|c|c|c|c|}
\hline $2-(v, b, r, k, \lambda)$ & Simple design $\mathcal{D}$ & $|\operatorname{Aut}(\mathcal{D})|$ & $\operatorname{Aut}(\mathcal{D})$ \\
\hline \hline $2-(7,28,16,4,8)$ & $2-(7,7,4,4,2)$ & 168 & $P S L(3,2)$ \\
$2-(15,30,16,8,8)$ & $2-(15,15,8,8,4)$ & 20160 & $A_{8}$ \\
$2-(10,30,18,6,10)$ & $2-(10,15,9,6,5)$ & 720 & $S_{6}$ \\
\hline
\end{tabular}

TABLE 17. BIBDs from the codes of fixed parts of orbit matrices for $Z_{4}$ acting on $T(10)$ and $T(14)$

\begin{tabular}{|c|c|c|}
\hline $2-(v, b, r, k, \lambda)$ & $|\operatorname{Aut}(\mathcal{D})|$ & $\operatorname{Aut}(\mathcal{D})$ \\
\hline \hline $2-(15,15,8,8,4)$ & 20160 & $A_{8}$ \\
\hline
\end{tabular}

ACKNOWLEDGEMENTS.

This work has been fully supported by Croatian Science Foundation under the project 1637 .

\section{REFERENCES}

[1] E. F. Assmus Jr. and J. D. Key, Designs and their Codes, Cambridge University Press, Cambridge, 1992, Cambridge Tracts in Mathematics, vol. 103 (second printing with corrections, 1993).

[2] M. Behbahani and C. Lam, Strongly regular graphs with non-trivial automorphisms, Discrete Math. 311 (2011), 132-144.

[3] T. Beth, D. Jungnickel and H. Lenz, Design Theory, Vol. I, Cambridge University Press, Cambridge, 1999.

[4] W. Bosma, J. Cannon and C. Playoust, The Magma algebra system. I. The user language, J. Symbolic Comput. 24 (1997) 235-265.

[5] A. E. Brouwer and C. J. van Eijl, On the p-rank of the adjacency matrices of strongly regular graphs, J. Algebraic Combin. 1 (1992), 329-346.

[6] A. E. Brouwer and J. H. van Lint, Strongly regular graphs and partial geometries, in: Enumeration and Design (eds. D. M. Jackson and S. A. Vanstone), Proc. Silver Jubilee Conf. on Combinatorics, Waterloo, 1982, Academic Press, Toronto, 1984, pp. 85-122.

[7] D. Crnković and S. Rukavina, Construction of block designs admitting an abelian automorphism group, Metrika 62 (2005), 175-183. 
[8] D. Crnković, M. Maksimović, B. G. Rodrigues and S. Rukavina, Self-orthogonal codes from the strongly regular graphs on up to 40 vertices, Adv. Math. Commun. 10 (2016), $555-582$.

[9] W. Fish, K. Kumwenda and E. Mwambene, Codes and designs from triangular graphs and their line graphs, Cent. Eur. J. Math. 9 (2011), 1411-1423.

[10] The GAP Group, GAP - Groups, Algorithms, and Programming, Version 4.8.10; 2018. (https://www.gap-system.org)

[11] W. H. Haemers, R. Peeters and J. M. van Rijckevorsel, Binary codes of strongly regular graphs, Des. Codes Cryptogr. 17 (1999), 187-209.

[12] M. Harada and V. D. Tonchev, Self-orthogonal codes from symmetric designs with fixed-point-free automorphisms, Discrete Math. 264 (2003), 81-90.

[13] Z. Janko, Coset enumeration in groups and constructions of symmetric designs, Combinatorics '90 (Gaeta, 1990), 275-277, Ann. Discrete Math. 52, North-Holland, Amsterdam, 1992.

[14] J. D. Key, J. Moori and B. G. Rodrigues, Permutation decoding for the binary codes from triangular graphs, European J. Combin. 25 (2004), 113-123.

[15] L. H. Soicher, The GRAPE package for GAP, Version 4.6.1, 2012, http://www.maths.qmul.ac.uk/ leonard/grape/

[16] V. D. Tonchev, Combinatorial Configurations: Designs, Codes, Graphs, Pitman Monographs and Surveys in Pure and Applied Mathematics 40, Wiley, New York, 1988.

\section{Kodovi iz orbitnih matrica jako regularnih grafova}

Dean Crnković, Marija Maksimović i Sanja Rukavina

SAŽETAK. U ovom članku pokazano je da uz određene uvjete podmatrice orbitnih matrica jako regularnih grafova razapinju samoortogonalne kodove. U svrhu prikaza ove metode konstruirani su samoortogonalni binarni linearni kodovi iz orbitnih matrica triangularnih grafova $T(2 k), k \leq 8$. Nadalje, pomoću riječi konstruiranih kodova dobiveni su jako regularni grafovi i blokovni dizajni.

Dean Crnković

Department of Mathematics

University of Rijeka

51000 Rijeka, Croatia

E-mail: deanc@math.uniri.hr

Marija Maksimović

Department of Mathematics

University of Rijeka

51000 Rijeka, Croatia

E-mail: mmaksimovic@math.uniri.hr

Sanja Rukavina

Department of Mathematics

University of Rijeka

51000 Rijeka, Croatia

E-mail: sanjar@math.uniri.hr

Received: 20.11.2017. 